\title{
Avaliação do Risco Nutricional de Pacientes Oncológicos Atendidos no Ambulatório da Unacon em um Hospital de Referência por meio da ASG-PPP
}

doi: https://doi.org/10.32635/2176-9745.RBC.2019v65n4.267

\author{
Evaluation of the Nutritional Risk of Oncological Patients Attended in the Outpatient of Unacon at a Reference Hospital \\ Through ASG-PPP \\ Evaluacion del Riesgo Nutricional de Pacientes Oncológicos Atendidos em la Clinica en un Hospital de Referencia a través \\ de ASG-PPP
}

\author{
Elenise da Silva Mota'; Regina Cely Marques Monteiro ${ }^{2}$; Keyce Lianne Siqueira Menezes ${ }^{3}$
}

\begin{abstract}
Resumo
Introduçáo: O câncer é uma doença crônica multicausal, caracterizando-se pelo crescimento desordenado, rápido e invasivo das células, alterando o seu material genético. Está associado a diversas alterações no trato gastrintestinal, podendo levar o paciente à desnutrição e à caquexia. Objetivo: Avaliar o risco nutricional, utilizando a Avaliação Subjetiva Global produzida pelo Próprio Paciente (ASG-PPP) de pacientes oncológicos em tratamento quimioterápico. Método: Estudo transversal, analítico, realizado com pacientes em tratamento quimioterápico atendidos no ambulatório com dados coletados da ASG-PPP. Resultados: Dos 78 pacientes analisados, a maioria era do sexo feminino $(68,82 \%)$ com idade entre $54 \pm 15$ anos. Os tumores prevalentes foram do esôfago e gástrico $(23,08 \%)$ e mama (23,08\%). As pacientes com tumor localizado na mama (52,38\%) apresentaram náuseas, vômitos e os odores provocavam enjoo. Observou-se que um percentual importante de pacientes relatou que estava comendo menos do que o normal, 44,83\% do sexo masculino e $32,64 \%$ do sexo feminino. $\mathrm{Na}$ análise do risco nutricional, o risco crítico foi recorrente entre os pacientes do estudo, sendo o grupo do tumor no rim o de maior participação (100\%). Não houve diferença significativa na avaliaçáo do diagnóstico nutricional $(\mathrm{p}<0,05)$, entretanto, foi verificado que grande parte dos pacientes apresentou algum tipo de necessidade de intervenção nutricional ao unir as três primeiras categorias. Conclusáo: A realização da avaliaçáo e do acompanhamento nutricional nos pacientes pode evitar ou minimizar os efeitos das alteraçóes decorrentes da doença, proporcionando um impacto positivo na qualidade de vida do paciente oncológico.

Palavras-chave: Avaliação Nutricional; Neoplasias/tratamento farmacológico.
\end{abstract}

\begin{abstract}
Introduction: Cancer is a chronic multicausal disease characterized by disordered, rapid and invasive growth of cells altering their genetic material. It is associated with several changes in the gastrointestinal tract and may lead the patient to malnutrition and cachexia. Objective: To evaluate the nutritional risk using the Patient-Generated Subjective Global Assessment (ASG-PPP) of cancer patients undergoing chemotherapy. Method: A cross-sectional, analytical study was conducted comparing chemotherapy treatments attended at the outpatient clinic with data collected from ASG-PPP. Results: Of the 78 patients analyzed, the majority were females $(68.82 \%)$ aged between $54+15$ years. The prevalent tumors were esophageal, gastric (23.08\%) and breast (23.08). Patients with a localized breast tumor $(52.38 \%)$ had nausea, vomiting and smells provoked sickness. A significant percentage of patients reported that they were eating less than normal $(44.83 \%)$ males and $(32.64 \%)$ females. In the nutritional risk analysis, the critical risk was recurrent among the study patients, with the kidney tumor group having the highest participation (100\%). There was no significant difference in the assessment of nutritional diagnosis $(\mathrm{p}<0.05)$, however, it was found that most patients needed some sort of intervention when the three first categories were gathered. Conclusion: The evaluation and nutritional monitoring in patients can avoid or minimize the effects of the changes resulting from the disease, providing a positive impact on the quality of life of cancer patients.

Key words: Nutrition Assessment; Neoplasms/drug therapy.
\end{abstract}

Resumen

Introducción: El cáncer es una enfermedad multicausal crónica caracterizada por un crecimiento celular desordenado, rápido e invasivo que altera su material genético. Se asocia con varios cambios en el tracto gastrointestinal y puede llevar al paciente a la desnutrición y la caquexia. Objetivo: Evaluar el riesgo nutricional mediante la Evaluación Subjetiva Global Producida por el Propio Paciente (ASG-PPP) de pacientes con cáncer que reciben quimioterapia. Método: Estudio analítico transversal realizado con pacientes sometidos a quimioterapia tratados en la clínica ambulatoria con datos recopilados de ASG-PPP. Resultados: De los 78 pacientes analizados, la mayoría eran mujeres $(68,82 \%)$ con edades entre 54 y 15 ańos. Los tumores prevalentes fueron esofágico y gástrico $(23,08 \%)$ y de mama $(23,08 \%)$. Las pacientes con un tumor mamario localizado $(52,38 \%)$ tenían náuseas, vómitos y los olores causaron náuseas. Un porcentaje significativo de pacientes informó que estaban comiendo menos de lo normal $(44,83 \%)$ hombres y $(32,64 \%)$ mujeres. En el análisis de riesgo nutricional, el riesgo crítico fue recurrente entre los pacientes del estudio, y el grupo de tumores renales tuvo la mayor participación (100\%). No hubo diferencias significativas en la evaluación del diagnóstico nutricional $(\mathrm{p}<0,05)$, sin embargo, se encontró que la mayoría de los pacientes tenían algún tipo de intervención nutricional cuando nos unimos a las tres primeras categorías. Conclusión: La realización de la evaluación y del seguimiento nutricional en los pacientes puede evitar o minimizar los efectos de las alteraciones resultantes de la enfermedad, proporcionando un impacto positivo en la calidad de vida del paciente oncológico.

Palabras claves: Evaluación Nutricional; Neoplasias/tratamiento farmacológico.

\footnotetext{
${ }^{1}$ Hospital Universitário João de Barros Barreto. Universidade Federal do Pará (UFPA). Belém (PA), Brasil. Orcid iD: https://orcid.org/0000-0003-0228-3406 ${ }^{2}$ Centro Universitário do Estado do Pará. Belém (PA), Brasil. Orcid iD: https://orcid.org/0000-0001-8959-1318 ${ }^{3}$ Centro Universitário do Estado do Pará. Belém (PA), Brasil. Orcid iD: https://orcid.org/0000-0001-5361-3116 Endereço para correspondência: Regina Cely Marques Monteiro. Travessa Mauriti, 1438 - Apto. 603 - Pedreira. Belém (PA), Brasil. CEP 66080-650. E-mail: nutrireginamarques@gmail.com
} 


\section{INTRODUÇÃO}

O câncer é considerado uma doença crônica não transmissível (DCNT), caracterizando-se por um crescimento desordenado das células ${ }^{1}$. Ocasiona alteraçóes catabólicas relevantes, podendo apresentar caquexia ${ }^{2}$. Atualmente, existem diversos tipos de modalidades convencionais de tratamentos antineoplásicos, entre eles, o mais efetivo é a quimioterapia, em virtude de apresentar maior incidência de curas de diversos tipos de tumores, inclusive os mais avançados, e tem revelado uma resposta positiva no que diz respeito ao aumento da sobrevida dos portadores de neoplasias ${ }^{3}$.

Em razão da sua toxicidade, a quimioterapia provoca efeitos adversos, como náuseas, vômitos, mucosite, constipação, diarreia, alteração no paladar, xerostomia e má absorção de nutrientes, podendo promover um quadro de desnutrição. Essa condição é associada a esse tipo de paciente em decorrência da baixa ingestáo de alimentos, alteraçôes metabólicas, morfológicas e funcionais causadas pelo tumor e do aumento da necessidade calórica por conta do crescimento tumoral ${ }^{3}$.

Visando a melhorar um possível estado de desnutrição, é imprescindível fazer a avaliação do estado nutricional do paciente oncológico precocemente e durante todo o tratamento, com o objetivo de identificar aqueles que apresentam risco nutricional, bem como os que já apresentam grau de desnutrição, para que possa ser realizada uma intervenção efetiva e imediata. Entre os métodos de triagem nutricional, está a Avaliaçáo Subjetiva Global produzida pelo Próprio Paciente (ASG-PPP), que é capaz de identificar a desnutrição antes mesmo do surgimento das alteraçóes na composiçáo corporal associada à falta de nutrientes ${ }^{4}$.

Este estudo teve por objetivo avaliar o risco nutricional em pacientes oncológicos em tratamento quimioterápico atendidos em um hospital de referência, por meio da utilização da ASG-PPP.

\section{MÉTODO}

Estudo transversal, analítico, com pacientes em tratamento quimioterápico atendidos no ambulatório da Unidade de Assistência de Alta Complexidade em Oncologia (Unacon) do Hospital João de Barros Barreto, em Belém-PA, no período de janeiro a agosto de 2018. A população analisada constituiu-se de 78 pacientes adultos e idosos, em tratamento quimioterápico, sendo que no grupo havia pacientes pré-cirúrgicos, pós-cirúrgicos e não cirúrgicos. Foram excluídos pacientes que não foram avaliados pela ASG-PPP e os que apresentaram prontuários com dados incompletos. A coleta de dados foi realizada após a aprovação do Comitê de Ética em Pesquisa do Hospital João de Barros Barreto, sob o CAAE: 01391418.5.0000.0017, de acordo com as Resoluçóes n. ${ }^{\circ}$ 466/2012 e n. ${ }^{\circ}$ 510/2016 do Conselho Nacional de Saúde, sobre Pesquisa envolvendo Seres Humanos, do Conselho de Saúde do Ministério da Saúde.

As variáveis sociais como sexo, idade, localização do tumor foram coletadas da ASG-PPP. Esse instrumento é um protocolo utilizado para triagem nutricional, sendo composto por duas partes: a primeira parte com sete itens; do item 1 ao item 4, as respostas eram dadas pelo próprio paciente e tratam de perguntas sobre o peso, ingestão alimentar, sintomas, atividades e função; já os itens 5 a 7 foram preenchidos pelo profissional da área da saúde, entre eles, o nutricionista. A somatória das caixas da parte preenchida pelo paciente e da parte preenchida pelo profissional da área da saúde representa o escore total da ASG-PPP e expressa as orientaçôes de triagem. O resultado obtido é escalonado da seguinte forma: de 0-1 (não necessita de intervenção no momento, mas deve ocorrer avaliação rotineira durante o tratamento); 2-3 (educação ao paciente e familiares por nutricionista ou outro profissional da área, com necessidade de intervenção farmacológica de acordo com os sintomas identificados); 4-8 (necessita de intervenção do nutricionista, juntamente com outros profissionais); $\geq 9$ (necessidade crítica de melhora no manuseio dos sintomas e/ou opçóes de intervenção nutricional). $\mathrm{O}$ resultado define o risco nutricional e as intervençôes nutricionais específicas a serem seguidas. A segunda parte do protocolo é respondida na sua integralidade pelo profissional de saúde e avalia o estresse metabólico do paciente, classificando-o em estados: estado A indica bem nutrido; estado B indica moderadamente desnutrido; e estado $\mathrm{C}$ representa o gravemente desnutrido. Neste estudo, foram analisados os itens relativos aos sintomas, à ingestão alimentar, ao risco nutricional e à avaliaçáo nutricional dos pacientes por meio dos dados fornecidos pela ASG-PPP.

Os tumores foram agrupados em cabeça e pescoço (localizados na cavidade oral, laringe, orofaringe e pescoço). Glândulas anexas (localizados no fígado, vesícula e pâncreas). Órgãos reprodutores femininos (localizados no útero e ovário). Tumores localizados no esôfago e estômago e o intestino (localizados no reto, cólon e intestino). Os outros tumores foram descritos isoladamente (mama, próstata, pulmão, rins e sarcoma de Kaposi).

As informaçóes foram registradas no Microsoft Office Excel 2013 e a análise realizada por meio do Software Bioestat 5.0 (Ayres, 2007), sendo adotado o nível de significância de $5 \%(p<0,05)$. Foi aplicado o teste exato de Fisher para comparação de proporçóes, visando a 
identificar uma possível diferença entre as categorias das variáveis avaliadas.

\section{RESULTADOS}

A população analisada foi composta de 78 pacientes oncológicos, sendo a maioria do sexo feminino $(62,82 \%)$ com média de idade obtida de $54 \pm 15$ anos e a idade mínima foi de 18 e máxima de 95 anos. Os tumores mais prevalentes foram os do esôfago e gástrico e o de mama, ambos com 18 pacientes $(23,08 \%)$. O tumor de rim e próstata foram os menos frequentes na amostra estudada (2,56\%), conforme os resultados descritos na Tabela 1 .

Em relação aos sintomas, a inapetência, descrita na ASG-PPP como sem apetite e rápido se satisfaz, foi mais recorrente nos pacientes com tumores localizados nos órgáos reprodutores femininos, alcançando 29,41\% desse grupo. Mais da metade das pacientes com tumor

Tabela 1. Categorização de pacientes oncológicos atendidos na Unacon, Belém-PA, 2018

\begin{tabular}{lcc}
\multicolumn{1}{c}{ Tipo de neoplasia } & n & $\%$ \\
\hline Cabeça e pescoço & 8 & 10,26 \\
Glândulas anexas & 7 & 8,97 \\
Mama & 18 & 23,08 \\
Órgãos reprodutores & 5 & 6,41 \\
Próstata & 2 & 2,56 \\
Pulmão & 5 & 6,41 \\
Rim & 2 & 1,28 \\
Sarcoma de Kaposi & 3 & 3,85 \\
Esôfago e gástrico & 18 & 23,08 \\
Intestino & 10 & 12,82 \\
Total & 78 & 100,00 \\
\hline
\end{tabular}

Fonte: Protocolo de atendimento nutricional - Hospital João de Barros Barreto, Belém-PA. localizado na mama apresentaram náuseas, vômitos e odores que enjoam $(52,38 \%)$, ao contrário dos pacientes com tumor de próstata que não apresentaram esse sintoma. Quase todos os pacientes referiram a presença de mais de um sintoma, independentemente da localização do tumor. Os sintomas mais frequentes foram náuseas, vômitos e odores que enjoam, enquanto os sintomas que menos apareceram foram diarreia ou constipaçáo, conforme apresentado na Tabela 2.

Examinando o consumo alimentar, a maioria dos pacientes não relatou mudanças, tanto os do sexo masculino $(48,28 \%)$ como os do sexo feminino $(42,86 \%)$. Entretanto, observou-se um percentual importante de pacientes que relatou que estava comendo menos do que o normal, sendo no sexo masculino $44,83 \%$ e no feminino $32,64 \%$. Foi observado que nenhum paciente estava fazendo uso da sonda ou de suplementos, resultados descritos na Tabela 3.

$\mathrm{Na}$ análise do risco nutricional, o risco crítico foi recorrente entre os pacientes do estudo, sendo o tumor localizado no rim o grupo com maior participaçáo (100\%), seguido dos pacientes com tumor no pulmáo $(83,33 \%)$ e daqueles que apresentaram tumor localizado no órgão reprodutor $(75 \%)$ e glândulas anexas $(71,43 \%)$. Os pacientes com sarcoma de Kaposi (66,67\%) necessitaram de intervenção conforme observado na Tabela 4.

Em relação ao estado nutricional, $65,52 \%$ do sexo masculino foram classificados com o estado nutricional $\mathrm{B}$, correspondente a moderadamente desnutridos ou sob risco de desnutrição, enquanto do sexo feminino, $46,94 \%$ apresentaram-se bem nutridos. Apesar de não ter sido observada diferença significativa nesses resultados $(\mathrm{p}<0,05)$, pode-se verificar que uma grande parte dos pacientes apresentou algum tipo de risco ou necessidade

Tabela 2. Sintomas de pacientes oncológicos atendidos na Unacon, Belém-PA, 2018

\begin{tabular}{|c|c|c|c|c|c|c|c|c|c|c|c|c|}
\hline \multirow[t]{2}{*}{$\begin{array}{c}\text { Tipo de } \\
\text { neoplasia }\end{array}$} & \multicolumn{2}{|c|}{$\begin{array}{c}\text { Sem apetite } \\
\text { e rápido } \\
\text { satisfaz }\end{array}$} & \multicolumn{2}{|c|}{$\begin{array}{c}\text { Náuseas, } \\
\text { vômitos e } \\
\text { odores que } \\
\text { enjoam }\end{array}$} & \multicolumn{2}{|c|}{$\begin{array}{l}\text { Diarreia ou } \\
\text { constipação }\end{array}$} & \multicolumn{2}{|c|}{$\begin{array}{c}\text { Xerostomia e } \\
\text { disgeusia }\end{array}$} & \multicolumn{2}{|c|}{$\begin{array}{c}\text { Disfagia, } \\
\text { odinofagia } \\
\text { e feridas na } \\
\text { boca }\end{array}$} & \multicolumn{2}{|c|}{ Total } \\
\hline & $\mathbf{n}$ & $\%$ & $\mathbf{n}$ & $\%$ & $\mathbf{n}$ & $\%$ & $\mathbf{n}$ & $\%$ & $\mathbf{n}$ & $\%$ & $\mathbf{n}$ & $\%$ \\
\hline Cabeça e pescoço & 2 & 11,76 & 4 & 23,53 & 3 & 17,65 & 4 & 23,53 & 4 & 23,53 & 17 & 100,00 \\
\hline Esôfago e gástrico & 8 & 23,53 & 9 & 26,47 & 4 & 11,76 & 6 & 17,65 & 7 & 20,59 & 34 & 100,00 \\
\hline Intestino & 3 & 20,00 & 3 & 20,00 & 1 & 6,67 & 5 & 33,33 & 3 & 20,00 & 15 & 100,00 \\
\hline Glândulas anexas & 4 & 25,00 & 5 & 31,25 & 2 & 12,50 & 4 & 25,00 & 1 & 6,25 & 16 & 100,00 \\
\hline Próstata & 1 & 25,00 & 0 & 0,00 & 1 & 25,00 & 1 & 25,00 & 1 & 25,00 & 4 & 100,00 \\
\hline Pulmão & 1 & 16,67 & 2 & 33,33 & 1 & 16,67 & 1 & 16,67 & 1 & 16,67 & 6 & 100,00 \\
\hline Órgão reprodutor & 5 & 29,41 & 4 & 23,53 & 2 & 11,76 & 4 & 23,53 & 2 & 11,76 & 17 & 100,00 \\
\hline Mama & 1 & 4,76 & 11 & 52,38 & 1 & 4,76 & 3 & 14,29 & 5 & 23,81 & 21 & 100,00 \\
\hline Rim & 2 & 25,00 & 2 & 25,00 & 2 & 25,00 & 1 & 12,50 & 1 & 12,50 & 8 & 100,00 \\
\hline
\end{tabular}

Fonte: Protocolo de atendimento nutricional - Hospital João de Barros Barreto, Belém-PA. 
de intervenção nutricional ao se unirem as três primeiras categorias: educação ao paciente, risco crítico e necessita de intervenção. Não houve diferença significativa quando se avaliou a classificação de risco entre os sexos, conforme os resultados descritos na Tabela 5 .

\section{DISCUSSÃO}

Neste estudo, foram excluídos pacientes que não foram avaliados pela ASG-PPP, bem como pacientes que apresentaram prontuários com dados incompletos. $\mathrm{O}$

Tabela 3. Alterações na ingestão alimentar de pacientes oncológicos atendidos na Unacon, Belém-PA, 2018

\begin{tabular}{|c|c|c|c|c|c|c|}
\hline \multirow{2}{*}{ Consumo alimentar } & \multicolumn{2}{|c|}{ Masculino } & \multicolumn{2}{|c|}{ Feminino } & \multicolumn{2}{|c|}{ Total } \\
\hline & $\mathbf{n}$ & $\%$ & $\mathbf{n}$ & $\%$ & $\mathbf{n}$ & $\%$ \\
\hline Sem mudanças & 14 & 48,28 & 21 & 42,86 & 35 & 44,87 \\
\hline Mais que o normal & 0 & 0,00 & 8 & 16,33 & 8 & 10,26 \\
\hline Menos que o normal & 13 & 44,83 & 16 & 32,65 & 29 & 37,18 \\
\hline Sólidos em menor quantidade & 6 & 20,69 & 10 & 20,41 & 16 & 20,51 \\
\hline Sólidos em pouca quantidade & 6 & 20,69 & 9 & 18,37 & 15 & 19,23 \\
\hline Apenas líquidos & 4 & 13,79 & 3 & 6,12 & 7 & 8,97 \\
\hline Apenas suplementos & 0 & 0,00 & 0 & 0,00 & 0 & 0,00 \\
\hline Muito pouco & 0 & 0,00 & 1 & 2,04 & 1 & 1,28 \\
\hline Alimentos pela sonda & 0 & 0,00 & 0 & 0,00 & 0 & 0,00 \\
\hline Total & 29 & 100,00 & 49 & 100,00 & 78 & 100,00 \\
\hline
\end{tabular}

Fonte: Protocolo de atendimento nutricional - Hospital Joáo de Barros Barreto, Belém-PA.

Tabela 4. Localização do tumor nos pacientes avaliados, segundo o risco nutricional, Belém-PA, 2018

\begin{tabular}{l|c|c|c|c|c|c|c|c|c|c|}
\hline \multirow{2}{*}{$\begin{array}{c}\text { Tipo de } \\
\text { neoplasia }\end{array}$} & \multicolumn{2}{|c|}{$\begin{array}{c}\text { Educação ao } \\
\text { paciente }\end{array}$} & \multicolumn{2}{c|}{ Em risco crítico } & \multicolumn{2}{c|}{$\begin{array}{c}\text { Necessita } \\
\text { intervenço }\end{array}$} & \multicolumn{2}{c|}{ Sem risco } & \multicolumn{3}{c}{ Total } \\
\cline { 2 - 13 } & $\mathbf{n}$ & $\%$ & $\mathbf{n}$ & $\%$ & $\mathbf{n}$ & $\%$ & $\mathbf{n}$ & $\%$ & $\mathbf{n}$ & $\%$ \\
\hline Cabeça e pescoço & 1 & 12,50 & 5 & 62,50 & 2 & 25,00 & 0 & 0,00 & 8 & 100,00 \\
Esôfago e gástrico & 1 & 5,56 & 12 & 66,67 & 4 & 22,22 & 1 & 5,56 & 18 & 100,00 \\
Intestino & 2 & 20,00 & 5 & 50,00 & 2 & 20,00 & 1 & 10,00 & 10 & 100,00 \\
Glândulas anexas & 2 & 28,57 & 5 & 71,43 & 0 & 0,00 & 0 & 0,00 & 7 & 100,00 \\
Próstata & 0 & 0,00 & 1 & 50,00 & 0 & 0,00 & 1 & 50,00 & 2 & 100,00 \\
Pulmão & 0 & 0,00 & 5 & 83,33 & 1 & 16,67 & 0 & 0,00 & 6 & 100,00 \\
Sarcoma de Kaposi & 1 & 33,33 & 0 & 0,00 & 2 & 66,67 & 0 & 0,00 & 3 & 100,00 \\
Órgão reprodutor & 0 & 0,00 & 3 & 75,00 & 1 & 25,00 & 0 & 0,00 & 4 & 100,00 \\
Mama & 4 & 22,22 & 5 & 27,78 & 9 & 50.00 & 0 & 0,00 & 18 & 100,00 \\
Rim & 0 & 0,00 & 2 & 100,00 & 0 & 0,00 & 0 & 0,00 & 2 & 100,00 \\
Total & 11 & - & 43 & - & 21 & - & 3 & - & 78 & 100,00 \\
\hline
\end{tabular}

Fonte: Protocolo de atendimento nutricional - Hospital Joâo de Barros Barreto, Belém-PA.

Tabela 5. Risco/diagnóstico nutricional de pacientes oncológicos, segundo o sexo, Belém-PA, 2018

\begin{tabular}{|c|c|c|c|c|c|c|}
\hline \multirow{2}{*}{ Consumo alimentar } & \multirow{2}{*}{ Categorias } & \multicolumn{2}{|c|}{ Masculino } & \multicolumn{2}{|c|}{ Feminino } & \multirow{2}{*}{$\mathbf{P}$} \\
\hline & & $\mathbf{n}$ & $\%$ & $\mathbf{n}$ & $\%$ & \\
\hline \multirow{5}{*}{ Risco nutricional } & Educação ao paciente & 3 & 10,34 & 8 & 16,33 & 0,5242 \\
\hline & Em risco crítico & 16 & 55,17 & 27 & 55,10 & 1.0000 \\
\hline & Necessita intervenção & 8 & 27,59 & 13 & 26,53 & 1,0000 \\
\hline & Sem risco & 2 & 6,90 & 1 & 2,04 & 0,5517 \\
\hline & Total & 29 & 100,00 & 49 & 100,00 & $\ldots$ \\
\hline \multirow{4}{*}{ Estado nutricional } & $\mathrm{A}$ & 7 & 24,14 & 23 & 46,94 & 0,0563 \\
\hline & B & 19 & 65,52 & 20 & 40,82 & 0,0692 \\
\hline & $\mathrm{C}$ & 3 & 10,34 & 6 & 12,24 & 1,0000 \\
\hline & Total & 29 & 100,00 & 49 & 100,00 & $\ldots$ \\
\hline
\end{tabular}

Fonte: Protocolo de atendimento nutricional - Hospital João de Barros Barreto, Belém-PA.

Nota: *Teste exato de Fisher: $p<0,05$ - diferenças significativas. 
Projeto Diretrizes Triagem e Avaliação do Estado NutricionaP afirma que a avaliaçáo é a primeira conduta a ser adotada para um atendimento nutricional de excelência, pois, por intermédio dela, o profissional imediatamente poderá detectar alteraçôes ligadas à nutrição e tomar decisôes de acordo com os resultados obtidos. Ainda acrescenta que a triagem e a avaliação são protocolos reconhecidos pelo Ministério da Saúde e tornaram-se obrigatórios para pacientes em atendimento hospitalar ou em ambulatórios. A utilização da ASG-PPP, incluindo itens referentes ao tumor e avaliados por uma pontuação numérica, possibilita identificar o risco nutricional do paciente e a necessidade de reavaliação. Com os dados incompletos, ou mesmo sem ter sido realizada a avaliação por meio desse método, pode haver recorrência de diagnósticos de risco nutricional deficitário ou incompleto. Desse modo, a ASG-PPP tornou-se, na prática clínica, um método de boa aceitabilidade reprodutibilidade e confiabilidade ${ }^{3}$.

No estudo que avaliou pacientes atendidos em um hospital de câncer no Maranhão, a prevalência foi de mulheres $(51,7 \%)$ com faixa etária acima de 59 anos $^{6}$, assim como neste estudo, com $62,82 \%$ de pacientes do sexo feminino com média de idade de 54+15 anos. Com relação à localização tumoral, o tumor de mama e do esôfago e gástrico foram os mais encontrados. Outros estudos demonstraram que os homens têm maior risco de desenvolverem câncer gástrico do que as mulheres e sua ocorrência aumenta com a idade ${ }^{7}$. Em outra pesquisa, em que foi avaliado o estado nutricional de 107 pacientes de ambos os sexos atendidos no ambulatório de um hospital no Distrito Federal, a maior incidência foi de mulheres, bem como o tipo de tumor foi o de mama ${ }^{8}$. Dados do Instituto Nacional de Câncer José Alencar Gomes da Silva (INCA $)^{9}$ revelaram que os tipos de câncer mais frequentes no mundo foram em primeiro lugar o de pulmáo e em segundo o de mama. Nos homens, o mais recorrente foi de pulmão $(16,7 \%)$ e nas mulheres foi o de mama (25,2\%). No Brasil, nas Regiōes Norte e Nordeste, o câncer de próstata e mama feminino foram dominantes, entretanto, os do colo do útero e de estômago começaram a apresentar relevância, sendo a Região Norte a única no país a ter as taxas dos cânceres de mama e do colo do útero em equivalência entre as mulheres.

Os sintomas na ASG-PPP são estratificados de acordo com o tipo de problema que possa impedir o paciente de comer de forma suficiente. Considerando os sintomas relatados com frequência pelos pacientes, a inapetência foi o mais descrito entre os pacientes de tumor nos órgáos reprodutores femininos. O estudo que avaliou 100 pacientes em uma Unidade de Oncologia e Hematologia em Porto Alegre demonstrou que os pacientes desnutridos foram os que mais apresentaram esse sintoma ${ }^{10}$.

Náuseas, vômitos e odores que enjoam foram os sintomas mais relatados, principalmente em pacientes com tumor localizado na mama (52,38\%). A náusea também foi o sintoma mais relatado em outra pesquisa ${ }^{4}$. A presença de náuseas e vômitos em pacientes em quimioterapia está relacionada com o tipo de droga prescrita, quantidade, via e velocidade de administração, ciclos recebidos e combinação com outras substâncias. Os quimioterápicos normalmente agem no retraimento da divisão celular. Como as células que revestem o estômago e o intestino possuem divisão mais acentuada e rápida, os quimioterápicos acabam por promover alterações negativas no revestimento epitelial. Dessa forma, as náuseas e vômitos podem ser frequentes em pacientes em tratamento ${ }^{11}$.

Os pacientes do estudo apresentaram todos os sintomas descritos na ASG-PPP. A maior frequência de sintomas foi encontrada nos pacientes com tumor de esôfago e gástrico. Esse fator pode afetar o estado nutricional e consequentemente demonstrar maior necessidade de assistência nutricional. Estudos indicaram que esse grupo de pacientes apresentou índice relevante de óbito hospitalar (cerca de 16,4\%), entendendo que é um público sensível a complicações nutricionais, em decorrência da localização do tumor ${ }^{12}$.

$\mathrm{Na}$ análise do consumo alimentar, foi observado um percentual importante de pacientes que relataram alguma alteração na ingesta alimentar, entre elas, redução na ingestão de sólidos e líquidos. Foi verificada também uma frequência de aproximadamente $45 \%$ de pacientes que não apresentaram alteração na ingesta alimentar. Resultado semelhante foi encontrado em uma análise de 83 pacientes oncológicos em quimioterapia atendidos no Hospital de Pelotas. Desses pacientes, $71,1 \%$ não apresentaram alteraçōes de ingestáo alimentar no momento da avaliação ${ }^{13}$. Em outras análises, a maioria dos pacientes da pesquisa apresentou ingestáo alimentar menor do que a normal $(46,47 \%)^{4}$. O mesmo ocorreu na avaliaçáo de 90 pacientes em tratamento quimioterápico, em que $31 \%$ apresentaram alteração de paladar e $30 \%$ perda de apetite. Os sintomas do trato gastrointestinal, aliados às drogas da quimioterapia, podem ser responsáveis por alteraçôes na mucosa da cavidade oral, provocando xerostomia, odinofagia e mucosite, o que reduz a ingestão alimentar ${ }^{14}$.

O risco nutricional é avaliado pela somatória da pontuaçáo dos escores da ASG-PPP e indica qual o tipo de intervençáo nutricional, sendo esse protocolo reaplicado novamente. Como a resposta é objetiva, indica se houve melhora ou regressão no estado nutricional. Dessa forma, possibilita a percepção de alteraçôes nutricionais e a imediata intervenção, viabilizando evitar que a desnutrição se instale gravemente e proporcionando uma melhor adesão ao tratamento. Na relação entre a localização do tumor e o risco nutricional, este estudo mostrou que quase metade dos pacientes estava em risco crítico e o tumor no esôfago e gástrico apresentou-se em maior número. 
O escore nutricional mais recorrente neste estudo foi o risco crítico, o qual foi relatado em outros estudos também como o mais frequente ${ }^{13,14}$. Mesmo em outros relatos nos quais os escores números encontrados estavam na média de 10,2 \pm 7 pontos, constatou-se que a intervenção nutricional prévia é fundamental para a prevenção e controle da desnutrição ${ }^{15}$.

$\mathrm{Na}$ relação entre o risco nutricional e o estado nutricional, este estudo, apesar de não apresentar diferença significativa nos resultados, mostrou que a maioria dos pacientes precisou de intervenção nutricional. Resultado semelhante verificou que mais da metade da amostra apresentou necessidade crítica de intervenção nutricional (escore $\geq 9$ ). Entretanto, ao agruparem os pacientes com escore de 4 a 8 , esse número elevou para $76,66 \%$, revelando a necessidade de intervenção nutricional ${ }^{4}$.

O estudo apresenta limitações. A heterogeneidade da população estudada com diferentes topografias e ampla faixa etária pode se tornar um viés. Outra limitação é o fato de terem sido excluídos pacientes que não foram avaliados pela ASG-PPP e os que apresentaram prontuários com dados incompletos, o que pode indicar que os dados coletados do prontuário contêm informaçóes omitidas no preenchimento, tornando o prontuário incompleto.

\section{CONCLUSÃO}

A avaliação nutricional é parte importante para o atendimento do paciente oncológico e a ASG-PPP é a ferramenta mais indicada para a realização dessa avaliação. Alem de ser um método pouco invasivo e de pouca complexidade, permite detectar precocemente os ricos nutricionais, bem como a intervençáo necessária de acordo com o estado do paciente. Neste estudo, avaliando o risco nutricional dos pacientes oncológicos em tratamento quimioterápico, utilizando a ASG-PPP, constatou-se que seus efeitos influenciaram o estado nutricional dos pacientes analisados. Os parâmetros nutricionais avaliados revelaram, em alguns tumores, alteraçóes significativas durante a referida terapia. Diante dos resultados encontrados, pode-se enfatizar a importância da avaliação e do acompanhamento nutricional adequado a esses pacientes, visando a evitar ou a minimizar a desnutrição, proporcionando um impacto positivo na qualidade de vida desses pacientes.

\section{CONTRIBUIÇÕES}

Os autores contribuíram igualmente de forma substancial em todas as etapas do trabalho e aprovaram a versão final a ser publicada.

\section{DECLARAÇÃO DE CONFLITO DE INTERESSES}

Nada a declarar.

\section{FONTES DE FINANCIAMENTO}

Não há.

\section{REFERÊNCIAS}

1. Instituto Nacional de Câncer José Alencar Gomes da Silva, Coordenação-Geral de Prevenção e Vigilância. Políticas e ações para prevenção do câncer no Brasil: alimentação, nutrição e atividade física. Rio de Janeiro: INCA; 2012.

2. Santos PI, Silva RGM, Monteiro TA, et al. A experiência do paciente com neoplasia: o significado da quimioterapia. REENVAP. 2012;(3):011-027.

3. Nascimento FSM, Góis DNS, Almeida DS, et al. A importância do acompanhamento nutricional no tratamento e na prevençáo do câncer. Caderno de Graduação-Ciências Biológicas e da Saúde-UNIT. 2015;2(3):11-24.

4. Gomes NS, Maia R. Avaliação subjetiva global produzida pelo próprio paciente e indicadores de risco nutricional no paciente oncológico em quimioterapia. Rev Bras Cancerol. 2015;61(3):235-42.

5. Sociedade Brasileira de Nutrição, Associação Brasileira de Nutrologia. Triagem e avaliaçáo do estado nutricional. São Paulo: Associação Médica Brasileira; 2011 (Projetos e diretrizes).

6. Santos AF, Lima FRS, Maciel MG, et al. Avaliação nutricional de pacientes com câncer gástrico e de outras localizaçōes. Rev Pesq Saúde. 2017;18(1):24-7.

7. Wachtel MS, Zhang Y, Chiriva-Internati M, et al. Different regression equations relate age to the incidence of Lauren types 1 and 2 stomach cancer in the SEER database: these equations are unaffected by sex or race. BMC Cancer. 2006;6:65. doi: https://doi. org/10.1186/1471-2407-6-65

8. Calado NPM, Cordeiro ALO, Fortes RC. Estado nutricional de pacientes oncológicos atendidos em hospital público do Distrito Federal. Rev. Bras Nutr Clin. 2016;31(2):142-8.

9. Instituto Nacional de Câncer José Alencar Gomes da Silva. Estimativa 2018: incidência de câncer no Brasil. Rio de Janeiro: INCA; 2017.

10. Ferreira D, Guimarães TG, Marcadenti A. Aceitação de dietas hospitalares e estado nutricional entre pacientes com câncer. Eisntein. 2013;11(1):41-6. doi: http:// dx.doi.org/10.1590/S1679-45082013000100008

11. Gozzo TO, Moysés AMB, Silva PR, et al. Náuseas, vômitos e qualidade de vida de mulheres com câncer 
de mama em tratamento quimioterápico. Rev Gaúcha Enferm. 2013;34(3):110-16. doi: http://dx.doi. org/10.1590/S1983-14472013000300014

12. Coruja MK, Steemburgo T. Estado nutricional e tempo de internação de pacientes adultos hospitalizados com diferentes tipos de câncer. Braspen J. 2017;32(2):114-8

13. Colling C, Duval PA, Silveira DH. Pacientes submetidos à quimioterapia: avaliação nutricional prévia. Rev Bras Cancerol. 2012;58(4):611-7.

14. Gabrielson DK, Scaffidi D, Leung E, et al. Use of an abridged scored Patient-Generated Subjective Global Assessment (abPG-SGA) as a nutritional screening tool for cancer patients in an outpatient setting. Nutr Cancer. 2013;65(2):234-9. doi: http://dx.doi.org/10.1080/0163 5581.2013.755554

15. Gonzales MC, Borges LR, Silveira DH, et al. Validação da versão em português da avaliação subjetiva global produzida pelo paciente. Rev Bras Nutr Clin. 2010;25(2):102-8. 